Military Technical College Kobry El-Kobba

Cairo, Egypt

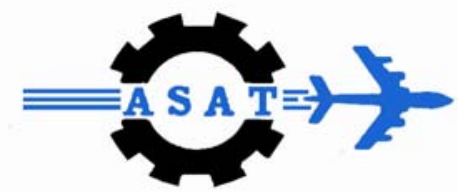

12-th International Conference

on

Aerospace Sciences \&

Aviation Technology

\title{
STUDY THE EFFECT OF QUADRATIC BLADE SHAPE ON THE VERTICAL AUTOROTATION PERFORMANCE OF A SMALL SCALE MODEL HELICOPTER
}

\author{
MOHAMED SUKRI M. A.* and ABAS A.W.*
}

\begin{abstract}
This paper presents the results of the numerical and experimental investigation of reducing the steady rate of descent of a typical small scale model helicopter. Power-off after hovering manoeuvre was picked for a case of study because of their critical induced velocity state. Contribution in every blade element in the effect of angle of attack, thrust and torque has been taken into consideration by blade element momentum theory for better results. In this analysis, test data of two types of blades with different driving region areas were obtained. The results of this study indicated that the expanding of the driving region area did reduce the steady rate of descent in vertical autorotation slightly but give lower rotor rotational speed compared to original shape blade. The study also had shown that zero collective pitch angle correction in the autorotation entry gives the best autorotation performance.
\end{abstract}

KEYWORDS: autorotation, driving region, rate of descent, quadratic blade

\footnotetext{
* Tutor, Dept. of Mechanical Eng., College Science and Technology, University of Technology Malaysia, City Campus Kuala Lumpur, Malaysia.sukri@citycampus.utm.my

^ Prof, Dr., Ir., Dept. of Aeronautical Eng., Faculty of Mech. Eng., University of Technology Malaysia, Skudai Malaysia. abas@fkm.utm.my
} 


\section{INTRODUCTION}

Autorotation is the continuous rotation of a body in an airstreams without the supply of external power [1]. The good example of autorotation phenomena is maple seed falling from the tree. Even though no power supply is provided, the body of maple seed keeps rotating. This is because airstreams from velocity descent was extracted by maple seed to provided sufficient power that needed. Because of that, descending velocity when helicopter power system failure is important feature to sustain rotor rotating, then make a safe landing. Indeed, understanding the factor influences the descent velocity is necessary.

Ideal autorotation is defined as steady rate of descent when the ideal power is zero. Therefore, the total vertical velocity components descend velocity and induced wake velocity in the rotor plane is zero [2]. However, in real autorotation the descent rate occurs at higher rate than ideal autorotation. This is because, profile power also taken into consideration. Additional of total power mean more kinetics energy is needed to balance the power consume by the rotor blade. Then, helicopter will descent at the higher steady rate where the velocity is sufficient to produce enough energy rate.

It is important to prevent main rotor rotation from decay rapidly. This is because, it produces very high torque from each blade [3] and rate of potential energy is not enough to provide sufficient kinetics energy to the rotor. The main factor to rotor decay is high torque from the high angle of attack. Because of that it is important to reduce collective pitch immediately after power failure. Upward velocity from descent velocity will increase angle of attack then retarding rotational speed with effect the total of main rotor thrust. Decreasing thrust will increasing rate of descent then this situation will continue till very high descent velocity. At this point no more rotational speed because the blades are completely stalled.

Helicopter performs glide after power-off generate additional energy needed to main rotor. This will decreasing steady rate of descent with increasing forward flight. In low region of forward flight, hover manoeuvre create the highest steady rate of descent [4]. This is because contribution of external power to the main rotor is purely rate of potential energy which is function of vertical velocity. Because of that improvement in vertical autorotation is believed benefit also to forward flight autorotation. 


\section{METHODOLOGY}

\subsection{Blade Elements Momentum Theory (BEMT)}

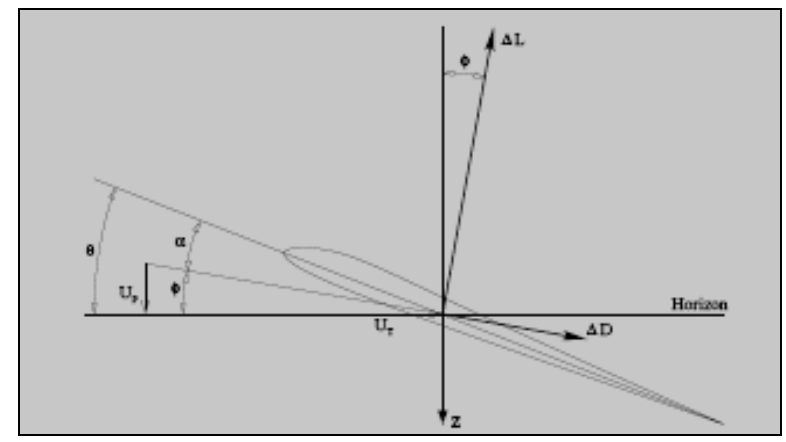

Fig.1 Incident velocities and aerodynamics environment at a typical blade element

Fig. 1 shows a sketch of the flow environment and aerodynamics forces at representative blade element on the rotor. The resultant velocity $U$ at the blade element $r$ is;

$$
\begin{aligned}
& U=\sqrt{U_{T}^{2}+U_{p}^{2}} \\
& U_{T}=V_{c}+v_{i} \\
& U_{p}=\Omega r
\end{aligned}
$$

The resultant incremental lift $\Delta L$ and drag $\Delta D$ per unit span on this blade element are;

$$
\begin{aligned}
& d L=\frac{1}{2} \rho U^{2}{ }_{c} C_{l} d r \\
& d D=\frac{1}{2} \rho U^{2} c C_{d} d r \\
& C_{l}=a \alpha \\
& C_{d}=\delta_{1}+\delta_{2} \alpha+\delta_{3} \alpha^{2}
\end{aligned}
$$

For NACA 0012 airfoil and low Mach number,

$$
\begin{aligned}
& a=5.75 \text { per radian } \\
& \delta_{1}=0.0087, \delta_{2}=-0.021, \delta_{3}=0.4
\end{aligned}
$$

The relative inflow angle $\phi$ and effective angle of attack $\alpha$ is;

$$
\begin{aligned}
& \phi=\tan ^{-1}\left(\frac{U_{P}}{U_{T}}\right) \\
& \alpha=\theta-\phi
\end{aligned}
$$


Therefore, the contributions to the thrust $\Delta T$, torque $\Delta Q$, and power $\Delta P$ of the rotor on this blade element are;

$$
\begin{gathered}
d T=N_{b}(d L \cos \phi-d D \sin \phi) \\
d Q=N_{b} r(d L \sin \phi-d D \cos \phi) \\
d P=N_{b} \Omega r(d L \sin \phi-d D \cos \phi)
\end{gathered}
$$

\subsubsection{Induced Velocity}

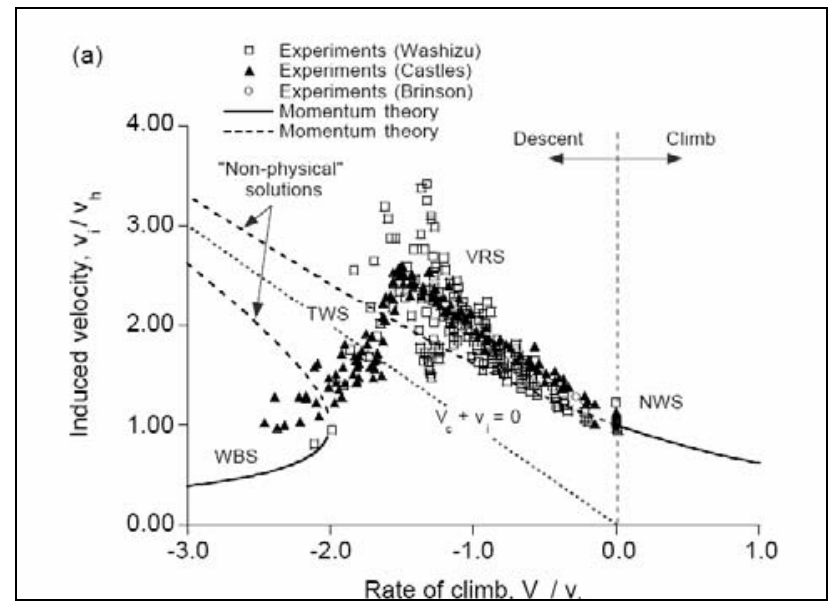

Fig. 2 Induced velocity curve by previous researcher [5, $6 \& 7]$

Figure 2 shows the complete induced velocity curve for a rotor in axial flight. The flight regimes of interest for the vortex ring state is for $-2 \leq v_{c} / v_{h} \leq 0$ where momentum based aerodynamic theories are strictly invalid. An approximation to the measured curve by Leishman [8] gives;

The region $-2 \leq V_{c} / V_{h} \leq 0$;

$$
v_{i}=1.15 v_{h}-1.125 v_{h}\left(\frac{v_{c}}{v_{h}}\right)-1.372 v_{h}\left(\frac{v_{c}}{v_{h}}\right)^{2}-1.718 v_{h}\left(\frac{v_{c}}{v_{h}}\right) 3-0.655 v_{h}\left(\frac{v_{c}}{v_{h}}\right)^{2} 4
$$

The region $v_{c} / v_{h} \leq-2$

$$
v_{i}=\left(\frac{-v_{c}}{2}\right)-\sqrt{\left(\frac{-v_{c}}{2}\right)^{2}-v_{h}^{2}}
$$

From momentum theorem hovering induced velocity $v_{h}$;

$$
v_{h}=\frac{-\frac{\Omega}{2} a c N_{b}+\sqrt{\left(\frac{\Omega}{2} a c N_{b}\right)^{2}+8 \pi N_{b} \Omega^{2} r a \theta c}}{8 \pi}
$$




\subsubsection{Dynamics Equation}

From Newtons second law, vertical force balance give;

$$
m a=m g-T+D
$$

From equation (4), (5) and (10) the total thrust $T$;

$$
T=\frac{1}{2} \rho U^{2} c N_{b} \int_{0}^{R}\left(C_{l} \cos \phi-C_{d} \sin \phi\right) d r
$$

The helicopter parasite drag $D$ is defined by an equivalent flat plate area $f$ as;

$$
D=\frac{1}{2} \rho\left(V_{c}+v_{i}\right)^{2} f
$$

Where the value of flat plate area [8] is approximately $f=1.2$

The torque balance equation can be expressed simply as;

$$
I_{R} \dot{\Omega}=-Q
$$

From equation (4), (5) and (11) the total torques Q;

$$
Q=\frac{1}{2} \rho U^{2} c N_{b} \int_{0}^{R}\left(C_{l} \sin \phi-C_{d} \cos \phi\right) r d r
$$

\subsubsection{Kinematical Relations}

The instantaneous velocity is defined as the limit of the average velocity as the time interval approaches zero. Thus;

$$
V=\lim _{\Delta t \rightarrow 0} \frac{\Delta S}{\Delta t}, a=\lim _{\Delta t \rightarrow 0} \frac{\Delta V}{\Delta t}
$$

Then, the rate of descent at time t can be express as:

$$
a d t=d V
$$

By using Euler method to this differential equation, the first order differential equation is:

$$
\frac{d y}{d t}=f(y, t)
$$

Then the method of numerical integration can be generalized to determine the function at $t=i+1$

$$
\begin{aligned}
& y_{i+1}=y_{i}+f\left(y_{i}, t_{i}\right) \Delta t \\
& t_{i}=i \Delta t
\end{aligned}
$$

Initial value constraints; 


$$
\left[\begin{array}{l}
v_{0} \\
y_{0}
\end{array}\right]=\left[\begin{array}{l}
0 \\
0
\end{array}\right],\left[\begin{array}{l}
\Omega_{0} \\
\theta_{0}
\end{array}\right]=\left[\begin{array}{l}
0 \\
0
\end{array}\right]
$$

Then the numerical solution becomes;

$$
\begin{aligned}
& {\left[\begin{array}{l}
v_{i+1} \\
y_{i+1}
\end{array}\right]=\left[\begin{array}{l}
\mathrm{v}_{\mathrm{i}}+f\left(\mathrm{v}_{\mathrm{i}}, \mathrm{y}_{\mathrm{i}}, \mathrm{t}_{\mathrm{i}}\right) \Delta \mathrm{t} \\
\mathrm{y}_{\mathrm{i}}+\mathrm{v}_{\mathrm{i}} \Delta \mathrm{t}
\end{array}\right]} \\
& {\left[\begin{array}{l}
\Omega_{i+1} \\
\theta_{i+1}
\end{array}\right]=\left[\begin{array}{l}
\Omega_{\mathrm{i}}+f\left(\Omega_{\mathrm{i}}, \theta_{\mathrm{i}}, \mathrm{t}_{\mathrm{i}}\right) \Delta \mathrm{t} \\
\theta_{\mathrm{i}}+\Omega_{\mathrm{i}} \Delta \mathrm{t}
\end{array}\right]}
\end{aligned}
$$

Where,

$$
\begin{aligned}
& f\left(\mathrm{v}_{\mathrm{i}}, \mathrm{y}_{\mathrm{i}}, \mathrm{t}_{\mathrm{i}}\right)=a_{i} \\
& f\left(\Omega_{\mathrm{i}}, \theta_{\mathrm{i}}, \mathrm{t}_{\mathrm{i}}\right)=\dot{\Omega}
\end{aligned}
$$

\subsection{EXPERIMENT}

The experimental set up for this project comprised of a model rotor, tower building, test

\begin{tabular}{|c|c|}
\hline SPECIFICATIONS & DIMENSION \\
\hline No. of Blade & 2 \\
\hline Airfoil & NACA 0012 \\
\hline Rotor diameter, $\mathrm{R}(\mathrm{m})$ & 0.6 \\
\hline Hub Weight, $M_{H}(\mathrm{~kg})$ & 0.316 \\
\hline One Blade Weight, $\mathrm{M}_{\mathrm{b}}(\mathrm{kg})$ & 0.077 \\
\hline Solidity, $\sigma$ & 0.053 \\
\hline Total mass moment inertia, i kg/m²) & 0.01 \\
\hline \multicolumn{2}{|l|}{ Cord Length, c (m) } \\
\hline 1) Rectangular Blade & 0.05 \\
\hline 2) Quadratics Blade & $\begin{array}{c}c=-\frac{0.12 r^{2}}{R^{2}}+\frac{0.12 r}{R}+0.03, r \text { is radial } \\
\text { distance }\end{array}$ \\
\hline
\end{tabular}
rig and electrical equipment and software for collecting, recording and analyzing data. The model rotor was equipped with controllable pitch system and having the specifications as shown in table 1 below.

Table 1: Model rotor specifications 


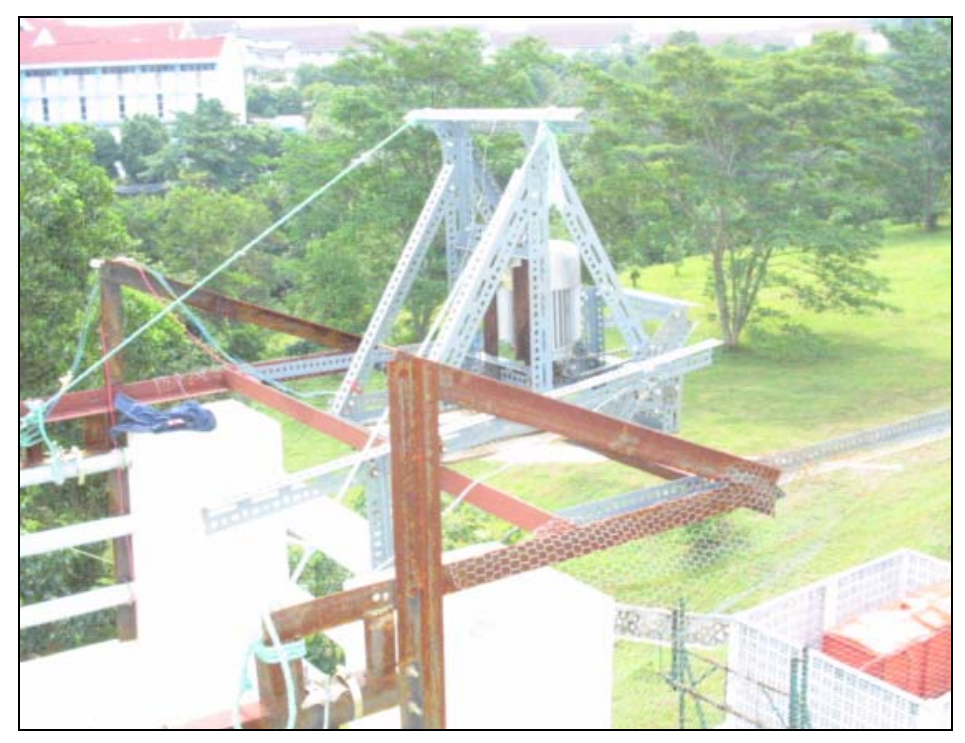

Fig. 3 Rig positioned on top of tower

The tower (Fig. 3) is 11.5 meter height in open area condition. The test rig consisting of an electric motor, safety net, sensor and rotor shaft was installed on the top of the tower. A guide wire from the centre of the shaft till the ground had been fixed vertically to prevent the blade from moving sideward. The ground end of the guide wire was fixed with spongy block to prevent the falling down blade from breaking. Video camera was used to measure the descent velocity. Photoelectric sensors had been used to count rotational speed of the model rotor along the drop test time. By using analogue to digital data acquisition system, LabView ${ }^{\circledR}$ user interface recorded and analyzed the data in the computer. The electrical schematics diagram and LabView ${ }^{\circledR}$ user interface are shown in Fig. 4 and 5 respectively.

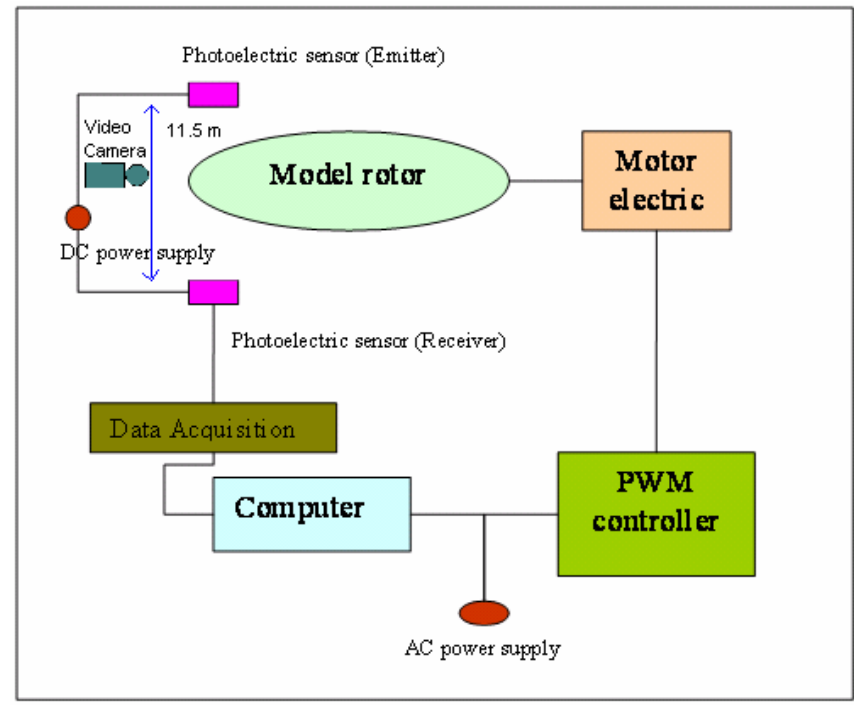

Fig. 4a Electrical schematic diagram 


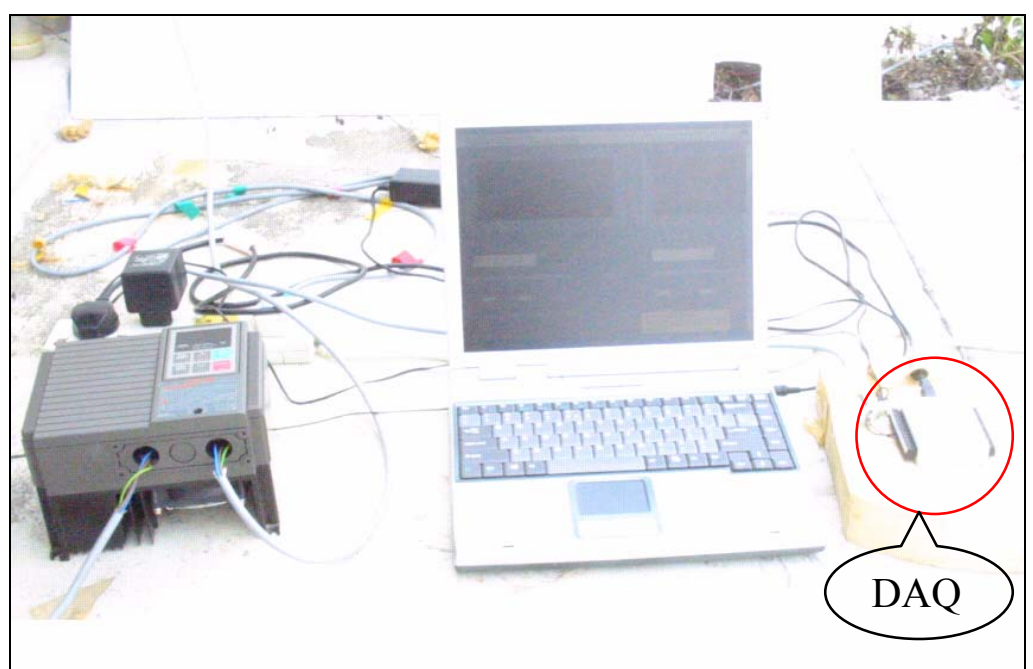

Fig. 4b DAQ connection on electrical system

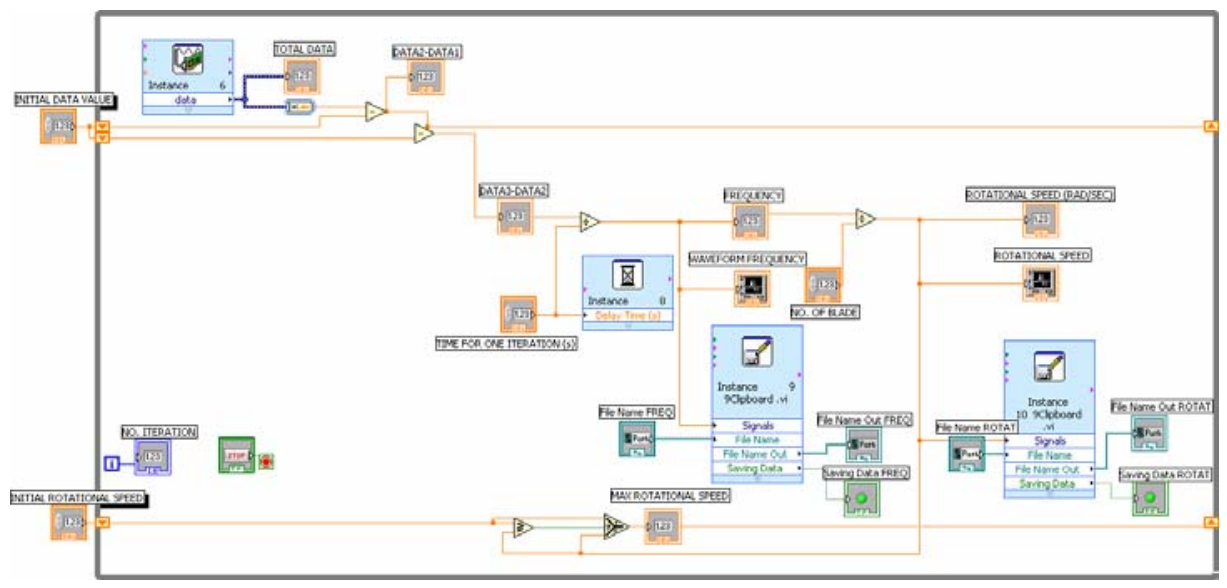

Fig. 5a LabView ${ }^{\circledR}$ user interface (block diagram)

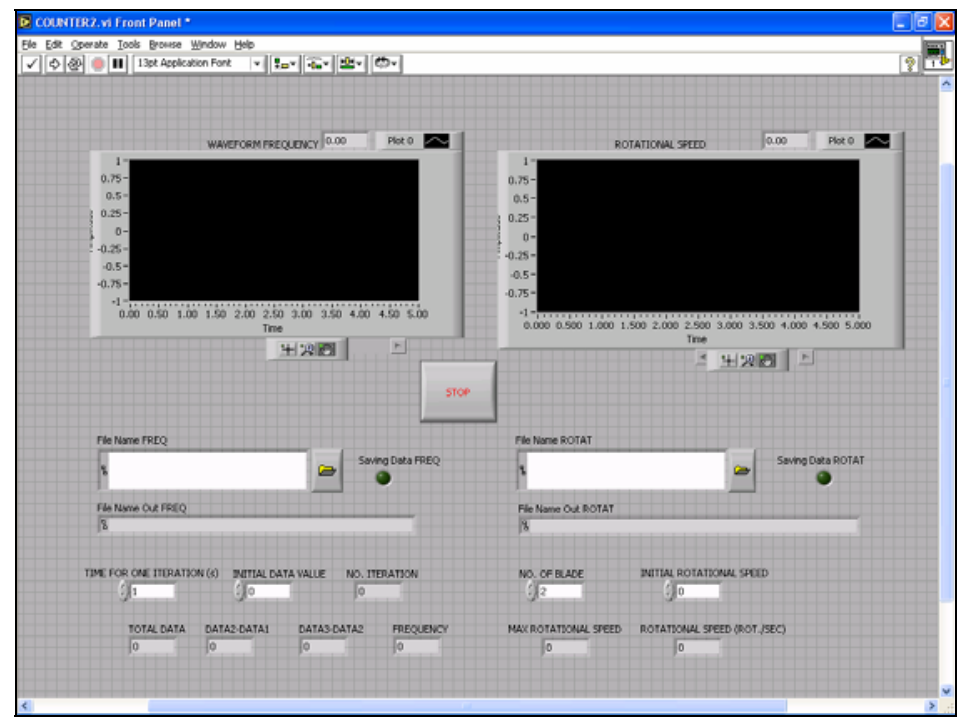

Fig. 5b LabView ${ }^{\circledR}$ user interface (front panel) 


\subsection{RESULTS AND DISCUSSION}

Lift distribution was important in order to make a safe landing by providing sufficient thrust. Therefore, the area with large positive lift force was preferred in the blade modification process. Also, to achieve steady rate of descent where the velocity of descent became constant, the lift distribution should become constant after at typical time. That means the lift distribution should be no longer change with time.

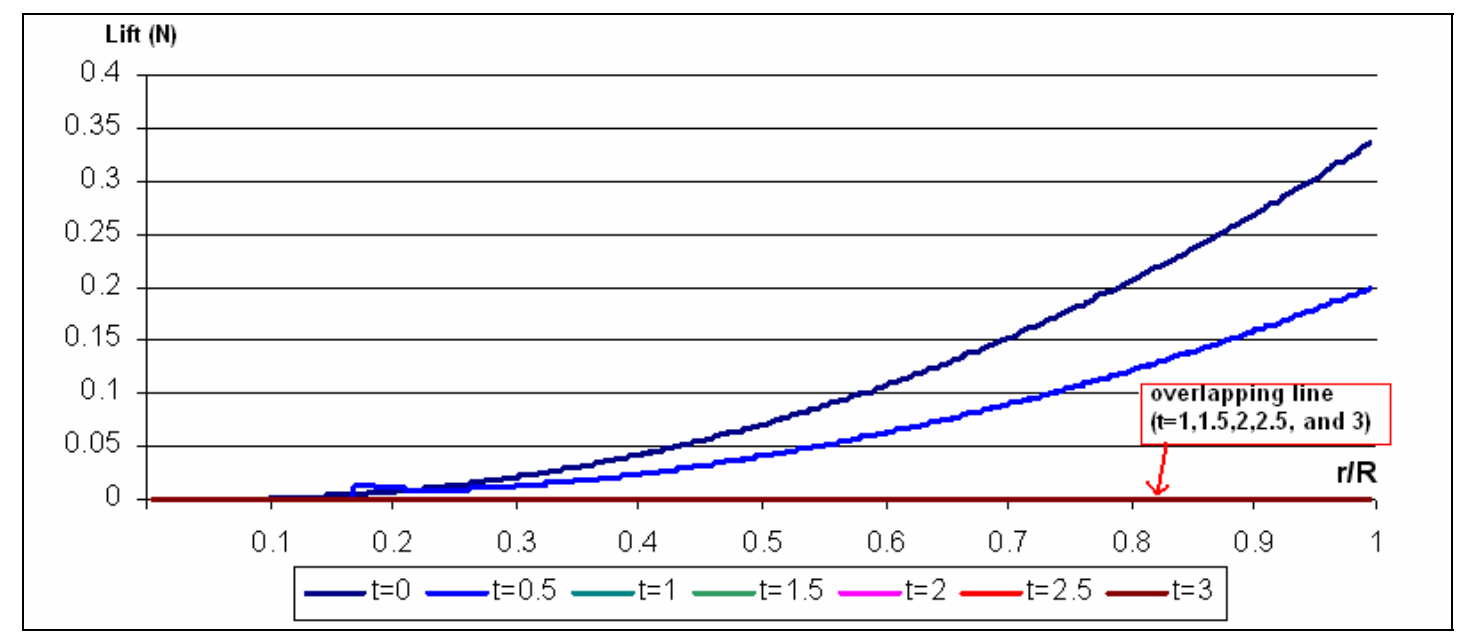

Fig. 6 Distribution of lift along the blade radius at hovering collective pitch

Figure 6 shows the lift distribution at the hovering collective pitch. During the initial flight maneuver, the lift was low at the root and increased to the maximum at the tip. For this two-bladed helicopter model, the summation of lift over all the blade sections was equal to half of the vehicle weight.

As the time increased, decreasing rotational speed had reduced the amount of lift produced. From the effects of both very low rotational speed and lift stall, the lift could not be produced at all the blade sections. Therefore at the hovering collective pitch, the velocity of descent was higher compared to at the lower collective pitch angle.

At zero collective pitch, the excessive decrease in rotational speed and stall phenomena that produced very low lift could be improved. Figure 7 shows that only at the initial condition the blade experienced completely negative lift and after that positive lift was developed. Then, the lift section line kept increasing with time and became constant at about 8 seconds flight maneuver. 


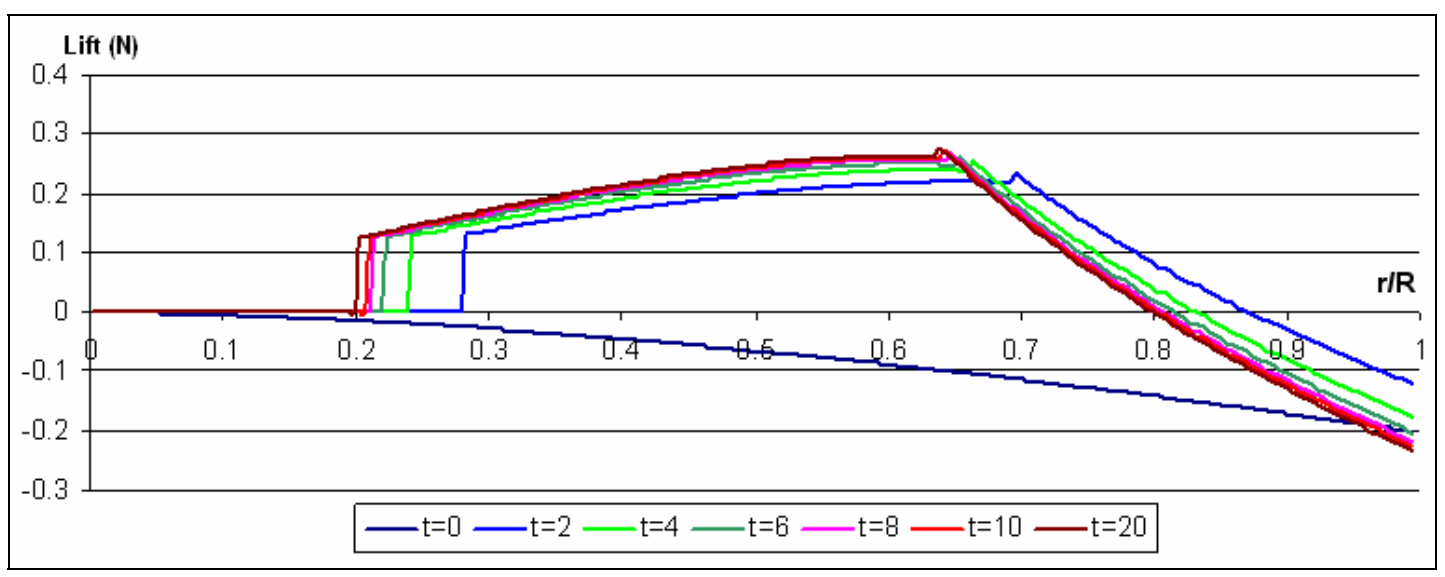

Fig. 7 Distribution of lift along the blade radius at zero collective pitch

The inboard area close to the root experienced blade stall and subsequently produced zero lift. Therefore, in Fig. 7 this region was represented by zero lift line. In the outboard area, negative lift was developed due to negative angle of attack. Between these two regions, positive lift was produced as the angle of attack was in the range of the stall angle and the zero angle of attack. This particular region played important role in determining the velocity of descent of the helicopter since it was the only region that contributed the helicopter's total thrust. Therefore in the blade modification for improving autorotation performance, this region should be greatly emphasized.

From Fig. 7, the driving region and the positive lift were located at the middle section of the blade. On the other side, the inboard and outboard regions developed decelerating torques and negative lift. Hence, it could be concluded that in an autorotation flight, the blade area could be divided into the favourable and adverse regions. Therefore, by optimizing or improving the area of these regions autorotation performance could be improved.

In the BEMT method, autorotation performances were assumed in the event of vortex ring state in ideal condition. Here, the flow state transition from vortex ring state to windmill brake state was only due to the magnitude of descent velocity. However, the degree of the unsteadiness of the vortex ring state was actually also depending on the type of rotor head, blade surface condition and wind gust. Therefore, in real condition the transition of the flow state from vortex ring state to windmill brake state also depends on these parameters.

Experimental methods were expected to give better autorotation performance results than the BEMT since many assumptions had been made in BEMT calculations. Therefore, this subsection is to investigate the helicopter model autorotation performances using both original and modified blades in the effect of real vortex ring state condition. The investigations were focused on the autorotation performance line trends, maximum or minimum line point value and performance comparison between these blades. As reference, free drop testing (Q0rpm) whereby no rotor rotation was applied had also been tested on the quadratic blade. 
Figure 8 shows the autorotation performances graphs at $-20^{\circ}$ collective pitch angle. As can be seen from the figure, the model helicopter had reached the ground from $11.5 \mathrm{~m}$ height in less than 2.5 seconds and for the QOrpm configuration the model helicopter had always reached at a typical point faster than others and reached the ground within $1.6 \mathrm{sec}$. For the cases of rectangular and quadratic blades, the model helicopter had reached the ground within $1.9 \mathrm{sec}$ and $2.0 \mathrm{sec}$ respectively. Therefore, analysis could only be made in between the time ranges.

Fig. 8 also shows that the plots for the velocity of descent are in the forms of typical quadratic equation graphs. The QOrpm configuration plot has its maximum velocity of descent of $9.5 \mathrm{~m} / \mathrm{s}$ at the drop time of $1.3 \mathrm{sec}$. The corresponding plot for rectangular blade has a lower maximum velocity of descent of $7.7 \mathrm{~m} / \mathrm{sec}$ occurring at fall time of $1.375 \mathrm{sec}$ while the plot for the quadratic blade has the lowest maximum velocity of descent of $2.3 \%$ less than that of the rectangular blade occurring at about $1.608 \mathrm{sec}$. Just as being predicted before, the QOrpm configuration will always give results of higher values than the others.

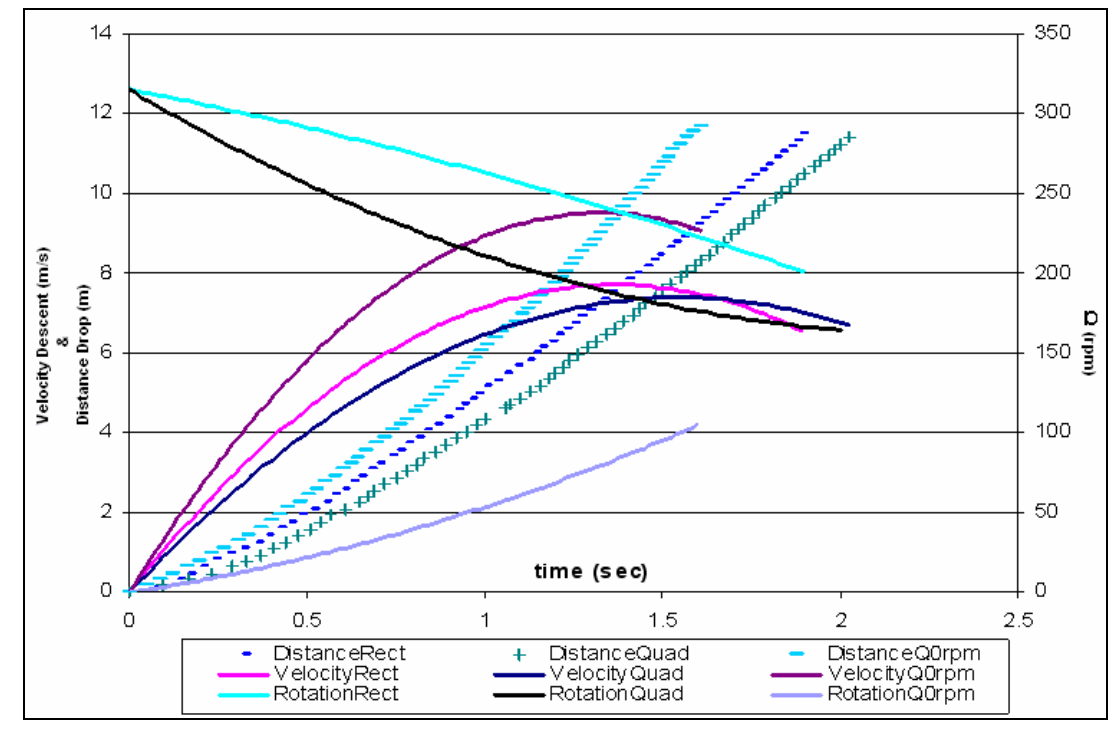

Fig. 8 Experimental results on autorotation performance for -20 deg pitch angle

For rotational speed performance, negative collective pitch setting had rotated the QOrpm rotor configuration from zero to about $100 \mathrm{rpm}$ in only $1.6 \mathrm{sec}$. In contrary, the rotational speed was reduced for both quadratic and rectangular blade configurations even though in the beginning the two configurations had been rotated at a hovering rotational speed. This was because when the initial rotational speed was very small or zero, vortex ring state could not be developed by the fact that free trailing vortex from each blade section dissipated away from the slipstream. Thus at a very small rotation, the flow around the rotor was in the windmill brake state having a low induced velocity 
and consequently producing both the inflow angle and angle of attack to create an accelerating torque region that rotated the rotor.

From Fig. 8, the difference in the autorotation performance between the different configurations was much obvious in terms of the rotor rotational speed rather than the velocity of descent. Also in this aspect, the quadratic blade showed a better performance than the rectangular one from the view point of the velocity of descent. The rotational speed of the quadratic blade configuration seems to have a tendency to become constant and becoming closer to that of the rectangular one after two second drop. It is predicted that both curves will be on top of each other a few second later. Therefore, autorotation performance of the rectangular blade configuration in term of rotational speed for this collective pitch angle was only good in the first few seconds.

Autorotation performance for zero collective pitch was depicted in Fig. 9. At this angle, QOrpm, rectangular blade and quadratic blade configurations reached the ground in $1.74,2.16$ and 2.15 seconds respectively. It was noted that the highest velocity of descent experienced by QOrpm configuration was about $10 \mathrm{~m} / \mathrm{sec}$ before becoming constant. For the rectangular and quadratic blade configurations their velocity of descent curves seemed to be close together up to $1.3 \mathrm{sec}$. Then after that the quadratic blade exhibited lower rate of descent. It is postulated that the velocity of descent for both the blade configurations that have the tendency to decrease will continue to decrease if much higher drop test was used.

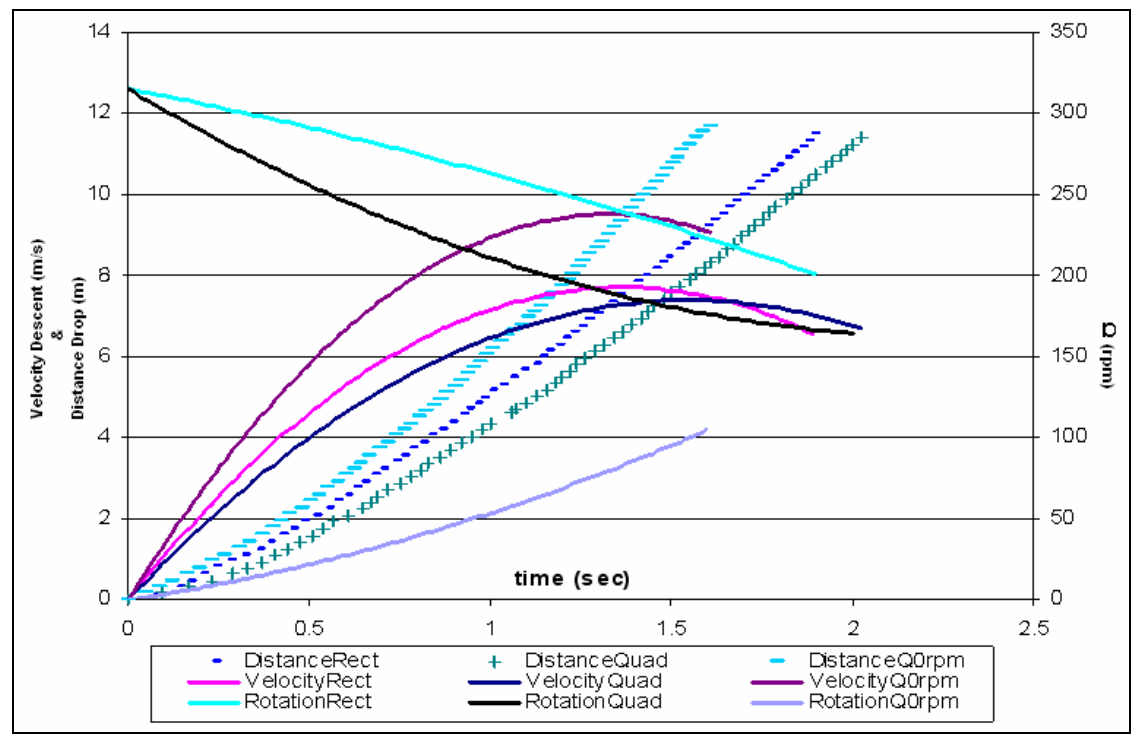

Fig. 9 Experimental results on autorotation performance for Odeg pitch angle

Also from the same figure, it is seen that the rotational speed for Q0rpm configuration had increased from zero to about $80 \mathrm{rpm}$. The reason for this happening is similar to the one being explained in the case when negative collective pitch angle was used. However because of the low negative inflow angle created by the zero pitch angle, the accelerating region was smaller compared to that of the high negative collective pitch 
setting. Consequently, the rotational speed that could be produced was also small. Nevertheless, the rectangular and quadratic blades seemed to give higher rotational speed as compared to QOrpm configuration. Furthermore, interestingly to note that the quadratic blade had always produced higher rotational speed than that of the rectangular one since the beginning of the autorotation manoeuvre.

Figure 10 shows the graph of autorotation performance for $10^{\circ}$ collective pitch setting. For all the three configurations, the time taken to reach the ground was not much different from the zero collective pitch. The times taken were 1.74, 2.14 and 2.26 seconds for the model helicopter with the QOrpm, rectangular and quadratic blades configurations to reach the ground respectively. The highest velocity of descent of 9.5 $\mathrm{m} / \mathrm{sec}$ was experienced by QOrpm configuration at $1.5 \mathrm{sec}$ after drop. The figure also shows that the velocities of descent for quadratic blade configuration were always lower than those of the rectangular one before becoming constant at $1.9 \mathrm{sec}$. Then at the steady rate of descent the quadratic blade configuration exhibited a $5 \%$ lower in descent velocity as compared to that of the rectangular blade configuration.

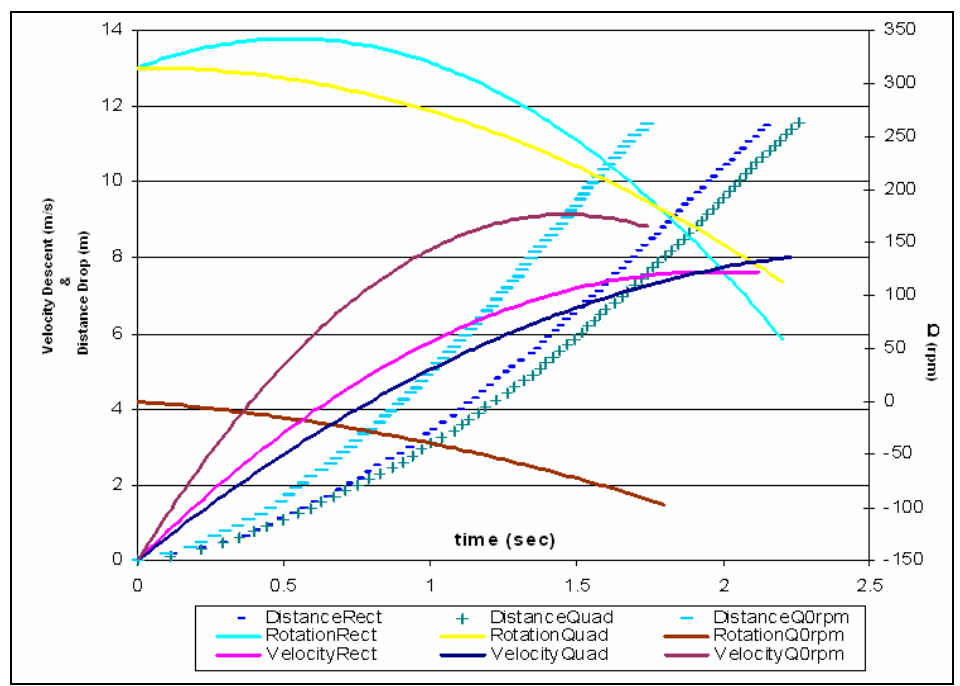

Fig. 10 Experimental results on autorotation performance for 10deg pitch angle

Reverse or negative rotational speed had also experienced by the QOrpm configuration at $10^{\circ}$ collective pitch angle. Increasing the collective pitch angle will increase the negative inflow angle and subsequently increase the positive angle of attack that leads to the existent of a large decelerating torque region. Hence, the decelerating torque that was more dominant than the accelerating torque made the QOrpm configuration to rotate in the reverse direction. In this particular condition, the QOrpm configuration was rotating up to about $100 \mathrm{rpm}$ in the reverse direction. However, through out the drop test the rectangular and quadratic blades configurations were still rotating in the normal direction of rotation. It is postulated that these two configurations will also experience some reverse direction rotation at the last stage of drop if the drop height was made higher than that of the existing one of $11.5 \mathrm{~m}$. 
Fig. 11 depicts the autorotation performance at a much higher collective pitch angle of $20^{\circ}$. At this pitch angle, the QOrpm configuration reached the ground at about $1.75 \mathrm{sec}$ after the drop. Both the rectangular and quadratic blades configurations reached the ground at about $1.8 \mathrm{sec}$ after drop. Also it can be seen from the figure, the plots of the velocity of descent for both the blade configurations were on top of each other. This was due to the high collective pitch angle causing the angle of attack to exceed the stall angle at the same total area along both the rectangular and quadratic blades. Hence, the total thrusts produced were equal in both blades configurations.

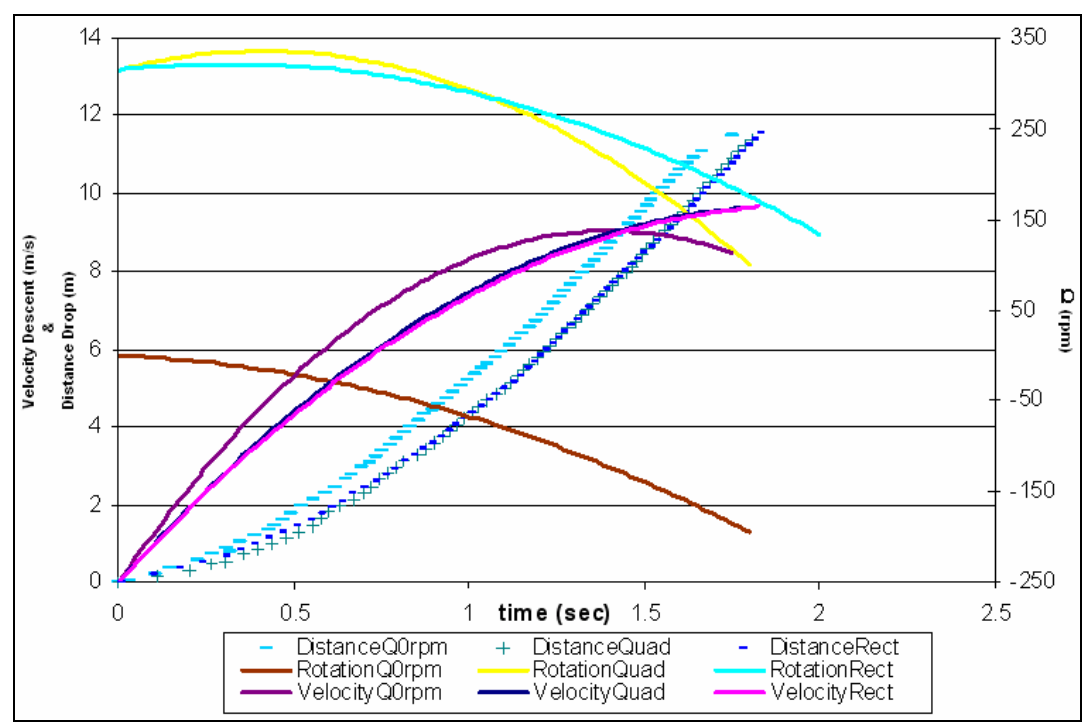

Fig. 11 Experimental results on autorotation performance for 20deg pitch angle

In this case, the QOrpm configuration also experienced reverse rotation. Since the high pitch angle caused the decelerating torque region to expand more, the magnitude of rotation achieved was higher than before. The figure also shows that the rotational speed for both the rectangular and quadratic blades configurations keeps decreasing and tends to reach the stage where the configurations will experience reverse rotation. Therefore, autorotation performances for $20^{\circ}$ collective pitch angle did not give good performance in both the descent velocity and rotational speed for all configurations.

\section{CONCLUSIONS}

From this research, it was shown that in a vertical autorotation flight the blade area could be divided into three sections namely the stall, driving and driven regions. These blade regions varied with the collective pitch angle setting and continuously changing until the steady autorotation being achieved. Also from this research, it was found that the driving region was necessary to keep the rotor speeding and produce enough thrust in the event of autorotation landing. Therefore, there was a possibility to improve autorotation performance by increasing the driving region area. 
In concluding, this research had shown that just a slight improvement in autorotation performance was obtained by using the quadratic blade. However, this situation is limited to zero collective pitch setting value only. Thus this shape of blade could give satisfactory improvement in the autorotation performance of the small scale model helicopter when collective pitch was lowering immediately to zero angle after the engine failure.

\section{Acknowledgment}

This research sponsored by University of Technology Malaysia.

\section{References}

[1] Skews, B. W., 1998. Autorotation of Polygonal Prisms with an Upstream Vane, Journal of Wind Engineering, 73: 145-158.

[2] Heyson, H. H., 1975. A Momentum Analysis of Helicopters and Autogyros in Inclined Descent with Comments on Operation Restrictions, NASA Technical Note, NASA TN D-7917

[3] Yeow, A., and Lee, N., 1985. Optimal Landing of a Helicopter in Autorotation. Master Thesis. Stanford University.

[4] Gessow, A., 1950. An Analysis of The Autorotative Performance of a Helicopter Powered by Rotor-Tip Jet Units, NASA Technical Note, NACA TN 2154

[5] Castles, W., Jr., and Gray, R.B., Empirical Relation between Induced Velocity, Thrust, and Rate of Descent of a Helicopter Rotor as Determined by Wind-Tunnel Test of Four Model Rotors. NASA Technical Report NASA TN-2474. October 1951.

[6] Washizu, K., Azuma, A., Koo, J, and Oka, T., Experiments on a Model Helicopter Rotor Operating in the Vortex Ring State. Journal of Aircraft. 1966. Volume (3): no (3): 225-230.

[7] Brinson, P., and Ellenrieder, T., Experimental Investigation of Vortex Ring Condition. Proceeding of the $24^{\text {th }}$ Annual European Rotorcraft Forum. September. Marseilles, France. 1998.

[8] Leishman, J.G. Principles of Helicopter Aerodynamics. Cambridge, United Kingdom: Cambridge University Press. 2000. 